\title{
Structural alignment using the generalized Euclidean distance between conformations
}

\author{
Ali R. Mohazab \\ Department of Physics and Astronomy, \\ University of British Columbia, Vancouver, BC, Canada \\ Steven S. Plotkin* \\ Department of Physics and Astronomy, \\ University of British Columbia, Vancouver, BC, Canada
}

${ }^{*}$ Corresponding author. Address: Department of Physics and Astronomy, University of British Columbia, 6224 Agricultural Road, Vancouver, BC V6T1Z1, Canada, Tel.: (604)822-8813, Fax: (604)822-5324, email: steve@phas.ubc.ca 


\begin{abstract}
The usual Euclidean distance may be generalized to extended objects such as polymers or membranes. Here this distance is used for the first time as a cost function to align structures. We examined the alignment of extended strands to idealized beta-hairpins of various sizes using several cost functions, including RMSD, MRSD, and the minimal distance. We find that using minimal distance as a cost function typically results in an aligned structure which is globally different than that given by an RMSDbased alignment.

Key words: Protein folding; Structural Alignment; RMSD; MRSD; Generalized distance ; Minimum distance; Reaction coordinate; order parameter; Optimization
\end{abstract}




\section{Introduction}

In a series of experiments starting in the late 1950s and culminating in a 1961 paper in the Proceedings of the National Academy of Sciences (2), C. B. Anfinsen showed that a protein such as bovine pancreatic ribonuclease would, under oxidizing conditions, undergo slow but spontaneous reshuffling of disulfide bonds from a state with initially random cross-linked pairs, to a state with correct disulfide pairing and full enzymatic activity. The spontaneous formation of correct disulfide pairs indicated that the amino acid sequence itself was guiding the process towards more thermodynamically favorable configurations, and the so-called thermodynamic hypothesis in protein folding was born.

This discovery underpinned the formalism developed decades later to understand protein folding as a configurational diffusion process on an energy landscape that through molecular evolution had the overall topography of a rugged funnel $(8,20,38,43,44$ ? ? ? ). The initial random crosslinkings and subsequent slow exchange of disulfide bonds observed by Anfinsen and colleagues argued against a mechanistic pathway picture, but there was nevertheless a lag phase before the energy landscape picture eventually took hold.

Though important as a conceptual tool, real predictive power was brought to bear by quantifying the funnel notion to generate free energy surfaces as a function of a progress coordinate that measured the degree to which a protein was folded $(9,45$ ? ). Soon thereafter, questions arose regarding what coordinate(s) best represented folding progress, or whether one could even find a simple geometric coordinate that would represent kinetically how folded a protein was. The kinetic proximity of a given configuration was quantified unambiguously as the probability a protein would fold first before unfolding, given that it was initially in that given configuration (19). This idea had earlier analogues in the Brownian analysis of escape and recombination probabilities of an ionized electron (37).

\section{Order parameters in protein folding}

The study of various order parameters that might best represent progress in the folding reaction have generated much interest $(5,66,7,11,12,15,17,18,19,22,27,29,32,46$ ? ? ), with questions focusing on what parameter(s) or principle component-like motions might best correlate with splitting probability or probability of folding before unfolding.

On the other hand, analyses using intuitive geometric order parameters have been developed to understand folding and are now commonly used. These include the fraction of native contacts $Q(7,13,15$, 28, 36, 42? ), which can be locally or globally defined, root mean square distance or deviation (RMSD) between structures $(23,26,51$ ? ), structural overlap parameter $\chi(4$, 14 ? ), Debye-Waller factors $(\underline{49}$, , 50), or fraction of correct Dihedral angles (28).

To find a simple geometrical order parameter that quantifies progress to the folded structure poses several challenges. These include an accurate account of the effects of polymer non-crossing (33), energetic and entropic heterogeneity in native driving forces $(31,41,42)$, as well as non-native frustration and trapping (16, 39, 47). Fortunately it has been borne out experimentally that wild type proteins are sufficiently minimally frustrated that non-native interactions do not play a strong role in either folding rate or mechanism, and native structure based models for folding rates and mechanisms have enjoyed considerable success $(1,3,21,35$ ? ? ).

In condensed matter systems, useful order parameters have historically had intuitive geometrical interpretations. Their definition did not require the knowledge of a particular Hamiltonian (although their temperature-dependence and time-evolution were affected by the energy function in the system). In chemical reactions, the distance between constituents in reactant and product has played a ubiquitous role in the construction of potential energy surfaces (30). Moreover from the point of view of stochastic escape and recombination, the distance perfectly correlates with the commitment probability for a freely diffusing particle between two absorbing boundaries. 


\section{Distance as an order parameter}

The distance is easy to define for a point particle, which we imagine to travel between two locations $A$ at $\mathbf{r}_{A}$ and $B$ at $\mathbf{r}_{B}$. It is the variational minimum of the functional:

$$
\int_{\mathbf{r}_{A}}^{\mathbf{r}_{B}} d s=\int_{0}^{T} d t \sqrt{\dot{\mathbf{r}}^{2}}
$$

where $\dot{\mathbf{r}} \equiv d \mathbf{r} / d t$, and the initial and final conditions, or equivalently boundary conditions, are $\mathbf{r}^{*}(0)=\mathbf{r}_{A}$ and $\mathbf{r}^{*}(T)=\mathbf{r}_{B}$.

However until recently $(33,34,40)$ the distance had not been formulated for higher dimensional objects such as pairs of polymer configurations, despite close parallels in string theory (? ).

In this paper after briefly reviewing two common reaction coordinates, Q and RMSD, and the two newer ones introduced and explored in (33, 34, 40), $\mathcal{D}$ and Mean root squared distance (MRSD), we will further explore structural alignments based on $\mathcal{D}$ for idealized hairpins.

\section{Some problems with commonly used reaction coordinates}

Many reaction coordinates have been used to describe the folding process, while still being flawed in principle. These characterizations have been largely successful because the majority of conformations during folding are well characterized by changes in these parameters: Proteins undergo some collapse concurrently with folding, lower their internal energy, and adopt structures geometrically similar to the native structure.

Nevertheless it is easy to point to simple examples of conformational transitions for which the adoption of native structure does not correlate with the change in commonly used order parameters. While these conformational pairs may not be wholly representative of the total folding process, they point to situations where folding to a given structure would not be well-characterized by commonly used order parameters.

Figure 1 shows two structures $A$ and $B$ with different measures of structural similarity to a "native" hairpin fragment $N$. These structures have different measures of proximity depending on the coordinate used to characterize them. If we use the fraction of native contacts $Q$ to describe native proximity *, structure $A$ has a $Q$ of $Q_{\mathrm{A}}=1 / 3$ while $Q_{\mathrm{B}}=0$, so by this measure it is more native. If we use the root

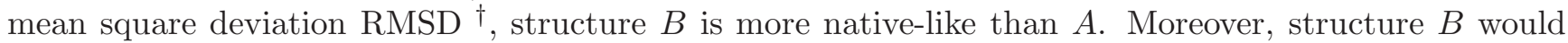
have a higher probability of folding before unfolding than $A$, i.e. it has a larger value of $p_{\mathrm{FOLD}}(19)$, and so is closer kinematically to the native structure. The longer the hairpin, the more likely a slightly expanded structure is to fold, so the discrepancy between $Q$ and $R M S D$ for these pairs of structures becomes even larger.

In contrast to $R M S D, Q$ also does not distinguish between chiralities. Typically the energy function forbids opposite chiralities, however if the appropriate chirality is not enforced in the backbone dihedral potentials, mirror-image structures as in figure 2 will be allowable, and are indistinguishable according to $Q(34)$.

While the RMSD is often characterized as a "distance" between structures, it is not equivalent or even proportional to the sum of the straight-line distances between the atoms or residues in the two structures (figure 3). This quantity is in fact given by the mean root squared distance (MRSD), defined for two

\footnotetext{
${ }^{*} Q_{\mathrm{AN}} \equiv\left(\sum_{i<j} \Delta_{i j}^{\mathrm{A}} \Delta_{i j}^{\mathrm{N}}\right) /\left(\sum_{i<j} \Delta_{i j}^{\mathrm{N}}\right)$ counts pairs of residues with some cutoff distance in both structure $A$ and structure $N$. This result is then normalized by the number of contacts in the native structure.

${ }^{\dagger} R M S D \equiv \sqrt{N^{-1} \sum_{i=1}^{N}\left(\mathbf{r}_{A_{i}}-\mathbf{r}_{B_{i}}\right)^{2}}$ is a least-squares measure of similarity between structures $A$ and $B$. Typically this quantity is minimized given two structures and so can be thought of as a "least squares fit". The sum may be over all atoms, or simply all residues in coarse-grained models.
} 
structures $A$ and $B$ as:

$$
\frac{1}{N} \sum_{n=1}^{N}\left|\mathbf{r}_{A_{n}}-\mathbf{r}_{B_{n}}\right|=\frac{1}{N} \sum_{n=1}^{N} \sqrt{\left(\mathbf{r}_{A_{n}}-\mathbf{r}_{B_{n}}\right)^{2}}
$$

The $R M S D$ between two structures is always greater than or equal to the $M R S D$ between the same structures, with $M R S D=R M S D$ in only the most trivial cases (34). The $R M S D$ is also less robust to large fluctuations of select residues in structural pairs (33).

MRSD has a simple intuitive physical meaning- the MRSD between two structures gives the average distance each residue in one structure would have to travel on a straight line to get to its counterpart in the other structure (fig 3).

\section{Polymer non-crossing in protein folding}

The above interpretation of $M R S D$ points to a shortcoming of both $M R S D$ and $R M S D$, which is the importance of chain non-crossing constraints. Consider the two curves depicted in fig 4 , which differ by having opposite sense of underpass/overpass. When both curves are aligned by minimizing $M R S D$ or $R M S D$, the respective values are almost zero. However the physically relevant distance for one conformation to transform to the other is much larger, and must involves one arm of the backbone circumventing the other as it moves between conformations. The transformation which minimizes the distance has been shown previously to involve motions wherein one end of the polymer doubles back upon itself until it reaches the underpass/overpass, where it appropriately crosses under/over it, and then proceeds snake-like to extend itself to the final position (33, 40). We will not deal further with the aspects of non-crossing in this paper.

\section{The generalized distance $\mathcal{D}$}

The distance between two points can be cast as a variational problem, where the arclength of the curve between two points is minimized (equation 1, see fig [5). The resultant Euler-Lagrange equations for the distance between two points are:

$$
\frac{d}{d t}\left(\frac{\partial \mathcal{L}}{\partial \dot{\mathbf{r}}}\right)=0
$$

or

$$
\dot{\hat{\mathbf{v}}}=\mathbf{0}
$$

which means straight line motion, since this means that the direction of the velocity does not change.

As mentioned in the introduction, the notion of distance between two points can be generalized to two curves or higher-dimensional objects in general (40). As in the case of points, the distance between two curves can be thought as a variational problem, where one now minimizes the cumulative integrated arclength between the two space curves:

$$
\mathcal{D}[\mathbf{r}]=\int_{0}^{L} d s \int_{0}^{T} d t \sqrt{\dot{\mathbf{r}}^{2}} .
$$

Here $\mathbf{r} \equiv \mathbf{r}(s, t)=(x(s, t), y(s, t), z(s, t))$ and $\dot{r} \equiv \partial \mathbf{r} / \partial t$. The independent variables in this formulation are position along the contour of the polymer $s$ and elapsed "time" during the transformation $t$.

Intuitively, the double integral in eq. (4) measures how much every part of the polymer moves in going from one configuration to another (see fig 6 for a schematic).

The minimal distance problem eq. (4) is not equivalent to a simple soap-film problem (see fig [7). It also has a lower symmetry than the relativistic world-sheet of a classical string (40), and so is inequivalent to that problem as well. 
Minimizing equation (4) results in straight line motion of all points along the curve. This is because equation (44) models not an inextensible string but an effective "rubber band" which can expand and contract at no cost to facilitate the minimal-distance transformation. If the polymer cannot arbitrarily stretch and contract (a good approximation for real polymers), the trajectories of the constituent segments deviate from straight lines.

The polymer is made inextensible by introducing the constraint

$$
\sqrt{\left(\frac{\partial \mathbf{r}}{\partial s}\right)^{2}} \equiv \sqrt{\mathbf{r}^{\prime 2}}=1
$$

whereupon the function to be minimized becomes

$$
\mathcal{D}=\int_{0}^{L} \int_{0}^{T} d s d t \mathcal{L}\left(\dot{\mathbf{r}}, \mathbf{r}^{\prime}\right)
$$

with effective Lagrangian:

$$
\mathcal{L}(s, t)=\sqrt{\dot{\mathbf{r}}}-\lambda\left(\sqrt{\mathbf{r}^{\prime 2}}-1\right) .
$$

and Lagrange multiplier $\lambda \equiv \lambda(s, t)$, a function of both $s$ and $t$.

The new equations of motion obtained by extremizing the functional become:

$$
\dot{\hat{\mathbf{v}}}=\lambda \boldsymbol{\kappa}+\lambda^{\prime} \hat{\mathbf{t}}
$$

where $\hat{\mathbf{t}}$ is the unit tangent vector and $\boldsymbol{\kappa}$ is the curvature vector (40).

Numerical solutions may be more readily obtained by discretizing the string as shown in figure 8. This procedure is a particular example of the method of lines, used to obtain solutions of partial differential equations. After discretization, the functional to be minimized becomes

$$
\mathcal{D}\left[\mathbf{r}_{i}, \dot{\mathbf{r}}_{i}\right]=\int_{0}^{T} d t \mathcal{L}\left(\mathbf{r}_{i}, \dot{\mathbf{r}}_{i}\right)
$$

where the effective Lagrangian $\mathcal{L}$ is:

$$
\mathcal{L}\left(\mathbf{r}_{i}, \dot{\mathbf{r}}_{i}\right)=\sum_{i=1}^{N}\left(\sqrt{\dot{\mathbf{r}}_{i}^{2}}-\frac{\lambda_{i, i+1}}{2}\left(\left(\mathbf{r}_{i+1}-\mathbf{r}_{i}\right)^{2}-b^{2}\right)\right) .
$$

Here $b$ is the segment length which we set to unity. The distances we obtain are thus in units of $b^{2}$. The distance between space curves has the dimensions of area just as the distance between points has dimensions of length. Upon discretization the PDE of the system becomes a set of $N$ coupled ODE's, one for each residue:

$$
\begin{aligned}
\dot{\hat{\mathbf{v}}}_{1}+\lambda_{12} \mathbf{r}_{2 / 1} & =0 \\
\dot{\hat{\mathbf{v}}}_{\mathbf{2}}-\lambda_{12} \mathbf{r}_{\mathbf{2} / \mathbf{1}}+\lambda_{23} \mathbf{r}_{3 / 2} & =0 \\
\vdots & \\
\dot{\hat{\mathbf{v}}}_{\mathbf{N}}+\lambda_{\mathrm{N}-\mathbf{1}, \mathbf{N}} \mathbf{r}_{\mathrm{N} /(\mathbf{N}-\mathbf{1})} & =0
\end{aligned}
$$

The solutions of the first and last $(N$ th) residues or beads consist of either straight-line motion of the bead, pure rotation of the link terminating on the bead, or a stationary solution where the residue remains at rest. Moreover, Weierstrass-Erdmann corner conditions or transversality conditions demand smooth curves for solutions by disallowing discontinuities or cusps in the trajectories (34).

Given two conformations which serve as boundary conditions on the equations of motion (11ar) 11c), 
several solutions yielding slightly (non-extensively) different $\mathcal{D}$ 's can be constructed. It can be shown that they are all local minima (34). In figure 9 two solutions are shown. Figure 9A depicts the global minimum transformation, and figure 9B a sub-minimal "excited-state" transformation. The solutions both involve either rotations of the constituent links or straight line motion of the constituent beads. In figure 9A, rotation occurs away from the straight-line conformation and results in a distance $\mathcal{D}=45.793$, while in 9B rotation occurs from the curved conformation and results in $\mathcal{D}=46.278$.

The fact that a real polymer cannot cross itself can be incorporated into the problem of finding the minimal distance (33). Non-crossing is manifested as an inequality constraint (10, 24, 25, 48), which appears in equation (10) as a Lagrange parameter for each residue $i$, multiplying the excluded volume constraint. To describe this, let the unit vector from the $k$ th to the $(k+1)$ th bead be $\hat{e}_{k} \equiv\left(\mathbf{r}_{k+1}-\mathbf{r}_{k}\right) / b$, then the vector to position $\mathbf{r}(s)$ at contour length $s$ on the chain (see e.g. fig $\mathbb{8}$ ) is

$$
\begin{aligned}
\mathbf{r}(s) & =b \sum_{i=0}^{k-1} \hat{e}_{i}+(s-k b) \hat{e}_{k} \\
& =\mathbf{r}_{k}+(s-k b) \hat{e}_{k} .
\end{aligned}
$$

To constrain the motion of the beads so that the chain cannot cross itself, we add the term

$$
l_{i}\left(\int_{0}^{s} d s\left|\mathbf{r}(s)-\mathbf{r}_{i}\right|+\epsilon_{i}^{2}\right)
$$

to the summand of equation (10). Note that by discretizing the problem to find the motion of residues, there must be an asymmetry in the way that the chain is treated- in a continuum treatment the term in the integrand of (12) would be $\left|\mathbf{r}(s)-\mathbf{r}\left(s^{\prime}\right)\right|$. The quantity $\epsilon^{2}$ in (12) is an "excess parameter" which is zero unless a residue is directly constrained (touching some part on the rest of the chain). If $\epsilon_{i}^{2}=0$ the problem of finding minimal distance is a "free" problem for residue $i$, and the equations of motion (11at 11c) are unchanged. However the corner conditions mentioned above induce an implicit "knowledge" of the sterically avoided boundary, so that the motion of the residues are altered to travel most directly to the steric surface constituting the constraint or obstacle. At this point the residue is constrained to be on the surface of the obstacle and the trajectory is defined accordingly. Subsequently the residue leaves the constraining surface and the problem becomes a free problem once again, travelling most directly to the final conformation (33).

In the above treatment the chain has zero thickness. A tube thickness $\rho$ can be straightforwardly incorporated into the treatment by letting $\mathbf{r}(s) \rightarrow \mathbf{r}(s)+\rho \hat{e}_{\rho}$ in equation (12), and then integrating over the surface of the cylinders which compose the resulting piece-wise tube.

Another modification that can be made to the Lagrangian is one involving the curvature constraints. In the current treatment the angle between to consecutive links of the chain can have any value, whereas in real protein chains angles defined by bonds between atoms or residues are restricted. We will not discuss these aspects in this manuscript.

\section{The minimal distance between protein fragments}

In ref. (33) protein fragments such as an alpha helix and beta hairpin were considered for purposes of calculating the minimal distance. An extended strand was aligned to the respective structures by minimizing either RMSD or MRSD, and the distance $\mathcal{D}$ was subsequently calculated for the aligned structural pairs. Both real and idealized protein fragments were considered. Most pairs of structures had smaller distance minimal pathways when aligned using MRSD as the cost function. In some cases however the smaller distance minimal pathway was obtained when the boundary conformations were aligned using RMSD as the cost function.

For example, the straight line conformation in figure 10 was aligned to an idealized $\beta$-hairpin structure 
also shown in that figure. The alignment was performed by both minimizing the MRSD between the structures (figure 10A), and by minimizing RMSD between the structures (figure 10B). In each instance, the minimal distance $\mathcal{D}$ between the structural pairs was calculated after alignment. The resulting aligned straight-line structures have significantly different position/orientation depending which cost function was used, MRSD or RMSD: the MRSD between the two staight-line structures is in fact larger than the MRSD between each and the hairpin structure (33).

Both transformations are minimal transformations but are subject to different boundary conditions and thus yield different pathways and $\mathcal{D}$ 's. The question remains as to how to align the structures to obtain the minimum of all minimal transformations, i.e. the minimum minimal distance $\mathcal{D}$. To calculate this quantity, $\mathcal{D}$ itself must be used as the cost function for alignment $t$

In this paper, we align structures using $\mathcal{D}$ as a cost function to obtain for the first time the minimum of all minimal transformations. The structures that we consider are idealized straight-line segments with varying number of links, which are then aligned to idealized beta hairpins using $\mathcal{D}$ as a cost function. The alignment and resulting distance $\mathcal{D}$ are compared with the alignments and distances of RMSD and MRSD. This is a first step toward aligning more complex structures using $\mathcal{D}$ as a cost function. We will also see that there exist high order approximations which capture much of the properties of a true $\mathcal{D}$ alignment. Applying these approximate metrics to align structures such as a full protein is a topic for future research.

\section{Structural alignment of protein fragments using the distance $\mathcal{D}$}

In principle, minimal pathways can be computed for any initial and final configurations, just as RMSD can be computed between any two configurations. However it of special significance to anneal the configurations allowing translations and rotations, until the minimal distance transformation is achieved (i.e. the minimum of minimal distance transformations). This is analogous to the usual procedure of using RMSD or MRSD as a cost function between two structures and minimizing with respect to translations and rotations. While the minimization procedure is particularly straightforward for RMSD and involves the inversion of a matrix, the minimization using the distance $\mathcal{D}$ as a cost function involves a simplex or conjugate gradient minimization and so is more computationally intensive.

In short the boundary conformations are allowed to translate and rotate in 3D space. Their position and orientation is modified to produce a pathway with minimal length, as compared to all other minimal pathways that can be obtained by positioning and orienting the same two structures in 3D space.

\footnotetext{
${ }^{\ddagger}$ In the limit of a large number of residues $(N)$, the distance converges to the $N$ times the $M R S D: \mathcal{D} \rightarrow N \times M R S D$, so for long chains MRSD can be considered a first step towards optimal alignment. But ideally one wants to align the two structures using $\mathcal{D}$ itself as a cost function.
} 


\section{Method and Results}

For the purpose of generating accurate initial guesses for the minimal distance aligned structure, we introduce the following hierarchy:

$$
\begin{aligned}
\mathcal{D}_{0} & =N \times M R S D \\
\mathcal{D}_{1} & =\sum_{i=1}^{N-1} \mathcal{D}\left(\ell_{i}^{(\mathrm{A})}, \ell_{i}^{(\mathrm{B})}\right) \\
\mathcal{D}_{2} & =\sum_{i=1}^{\operatorname{int}((N-1) / 2)} \mathcal{D}\left(\left\{\ell_{i}^{(\mathrm{A})}\right\},\left\{\ell_{i}^{(\mathrm{B})}\right\}\right)+\mathcal{D}_{1}^{(\text {end link })} \\
\vdots & \\
\mathcal{D}_{N} & =\mathcal{D} .
\end{aligned}
$$

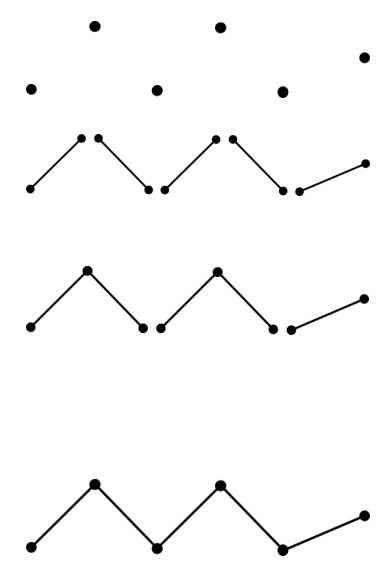

In this hierarchy, the $\mathcal{D}_{\alpha}$ have the following interpretation: $\mathcal{D}_{0}$ is the cumulative distance between the sets of points comprising the residue locations of conformations $A$ and $B, \mathcal{D}_{1}$ is the cumulative distance between the sets of single links, $\ell_{i}$, comprising configurations $A$ and $B, \mathcal{D}_{2}$ is the cumulative distance between the sets of double links, $\left\{\ell_{i}\right\}$, comprising configurations $A$ and $B$ plus any single-link remainder if one exists, and so on. That is, at level $\alpha$ the polymer chain is divided up into sub-segments each of link-length $\alpha$, plus one segment constituting the remainder. When $\alpha=N$, the chain as a whole is considered, which is the true distance $\mathcal{D}$. This procedure is also illustrated schematically adjacent to each equation above.

We observed that $\mathcal{D}_{1}$ was a good approximation to the total $\mathcal{D}$ between two chains, was much easier in practice to calculate, and could be automated in a robust way. For these reasons we used it to generate initial guesses for minimal distance aligned structures. After the initial alignment using $\mathcal{D}_{1}$ the chains were further aligned using the full distance $\mathcal{D}$. At this stage the general form of the transformation is established and the computation can be automated. We used a Nelder-Mead simplex method in our algorithm to find the minimal distance alignment.

Figure 11 shows the aligned structures using $R M S D, M R S D, \mathcal{D}_{1}$, and $\mathcal{D}$, for increasing numbers of links. Several points can be observed. For the smallest number of links $(3), M R S D$, $\mathcal{D}_{1}$, and $\mathcal{D}$ all give the same alignment (fig [1]a). For 5 or more links, the $M R S D$-aligned structure breaks symmetry by choosing particular diagonal direction, while $\mathcal{D}_{1}$ and $\mathcal{D}$ retain this symmetry but begin to differ (fig $11 \mathrm{~b}$ ). The deviation from $M R S D$ and $\mathcal{D}$ is a finite-size effect (40), so we know that the two alignments must eventually converge as $N$ is increased. At 9 links (fig $11 \mathrm{~d}$ ), the $\mathcal{D}_{1}$-alignment breaks symmetry in the same fashion as MRSD, yet the $\mathcal{D}$-alignment remains similar to RMSD. By 11 links (fig 11k), the $\mathcal{D}$ aligned structure has broken symmetry as well, however with a smaller angle to the horizontal than either $M R S D$ or $\mathcal{D}_{1}$. As $N$ is increased, $\mathcal{D}_{1}$ and $M R S D$ aligned structures quickly converge, while the angle with respect to the horizontal of the $\mathcal{D}$-aligned structure continues to lag behind that of either $M R S D$ and $\mathcal{D}_{1}$ structures, converging slowly as $N$ continues to increase (figures [11- $\mathrm{j}$ ). The RMSDaligned structure remains horizontal throughout.

Average lengths of $\beta$-hairpins in databases constructed from the PDB are about 17 residues (? ), most consistent with fig 11 h. From this figure we see that hairpins of this length have a globally different structural alignment with extended structures depending on whether $\mathcal{D}$ or $R M S D$ is used.

Table 1 and figure 12 summarize the results for the minimal distance transformations from the aligned structures. Table 1 gives the numerical value of the distance $\mathcal{D}$ for each aligned structure, aligned using the various cost functions listed: $\mathcal{D}, \mathcal{D}_{1}, M R S D$, and $R M S D$. Note that the distance $\mathcal{D}$ is always minimized for the distance-aligned structure, and tends to increase as one considers the $\mathcal{D}_{1}, M R S D$ and 
then $R M S D$-aligned structures for a given number of links.

For comparison, in table 2 the corresponding values of MRSD are given for the aligned structures using each cost function. Note in each table that as $N \rightarrow \infty, \mathcal{D}$ tends to converge to MRSD.

The distance travelled per residue, in units of link length is $\mathcal{D} / N b$. Dividing this measure by the chain length $(N-1) b$ gives a scale-invariant measure of the distance: $\tilde{\mathcal{D}}=\mathcal{D} /\left(N(N-1) b^{2}\right)$. This quantity is plotted in figure 12. We can see from the plot that the $D_{1}$-aligned structure generally gives a good approximation to the true $\mathcal{D}$-aligned structure. Moreover, $M R S D, \mathcal{D}_{1}$ and $\mathcal{D}$ all converge to the same while RMSD converges to a dissimilar value.

\section{Conclusion and Discussion}

In this paper, we reviewed the concept of the generalized distance $\mathcal{D}$, and then used it as a cost function to align unfolded idealized strands of various sizes to their corresponding idealized $\beta$-hairpin structures. This is the first time that the true Euclidean distance has been used as a cost function for structural alignment. The distance $\mathcal{D}$ for the minimal transformation between aligned structural pairs was compared for various alignment cost functions: $R M S D, M R S D, \mathcal{D}_{1}$, and $\mathcal{D}$ itself. $\mathcal{D}_{1}$ is the distance between conformational pairs if the chain were decimated to single links and distance of all single-link transformations was summed.

We found that $\mathcal{D}_{1}$-aligned structures generally gave a distance that was close to the true $\mathcal{D}$-aligned structure, and in this sense was a good approximation. However the aligned structures were noticeably different depending on the cost function, for the finite values of $N$ that we studied. Our largest value of $N$ was 22 residues, while the average length of $\beta$-hairpins is about 17 residues. For these average hairpin lengths, the minimal $\mathcal{D}$ aligned structure is globally different from the $R M S D$ structure. Whether this discrepancy is generally true for larger structures or whole proteins remains to be determined, but we feel it is likely. It is not yet clear at this point whether alignment using distance will yield more accurate predictions for such problems as protein structure prediction or ab-initio drug design. What is clear is that the best-aligned structures using a reasonable alignment metric such as the true distance give very different results than RMSD, even for relatively simple structures such as the beta-hairpin.

\section{Acknowledgements}

S.S.P. gratefully acknowledges support from the Natural Sciences and Engineering Research Council, and the A. P. Sloan Foundation.

\section{References}

1. E. Alm and D. Baker. Prediction of protein-folding mechanisms from free-energy landscapes derived from native structures. Proc Nat Acad Sci USA, 96:11305-11310, 1999.

2. C. B. Anfinsen, E. Haber, M. Sela, and F.H. White, Jr. The kinetics of formation of native ribonuclease during oxidation of the reduced polypeptide chain. Proc Nat Acad Sci USA, 47:1309, 1961.

3. D. Baker. A surprising simplicity to protein folding. Nature, 405:39-42, 2000.

4. A. Baumketner and Y. Hiwatari. Diffusive dynamics of protein folding studied by molecular dynamics simulations of an off-lattice model. Phys. Rev. E, 66(1):011905, Jul 2002.

5. A. Baumketner, J.-E. Shea, and Y. Hiwatari. Improved theoretical description of protein folding kinetics from rotations in the phase space of relevant order parameters. J. Chem. Phys., 121(2):11141120, 2004. 
6. David A. C. Beck and Valerie Daggett. A One-Dimensional Reaction Coordinate for Identification of Transition States from Explicit Solvent Pfold-Like Calculations. Biophys. J., 93(10):3382-3391, 2007.

7. Robert B. Best and Gerhard Hummer. Reaction coordinates and rates from transition paths. Proc Nat Acad Sci USA, 102((19)):6732-6737, 2005.

8. J. D. Bryngelson, J. N. Onuchic, N. D. Socci, and P. G. Wolynes. Funnels, pathways and the energy landscape of protein folding. 21:167-195, 1995.

9. Joseph D. Bryngelson and Peter G. Wolynes. Intermediates and barrier crossing in a random energy model (with applications to protein folding). J Phys Chem, 93(19):6902-6915, 1989.

10. D. Cass. Optimum growth in an aggregative model of capital accumulation. Rev. Econ. Stud., 32:233-240, 1965.

11. Hue Sun Chan and Ken A. Dill. Transition states and folding dynamics of proteins and heteropolymers. J Chem Phys, 100(12):9238-9257, 1994.

12. L. L. Chavez, J. N. Onuchic, and C. Clementi. Quantifying the roughness on the free energy landscape: Entropic bottlenecks and protein folding rates. J Am Chem Soc, 126:8426-8432, 2004.

13. Margaret S. Cheung, Angel E. Garcia, and Jose N. Onuchic. Protein folding mediated by solvation: Water expulsion and formation of the hydrophobic core occur after the structural collapse. Proc. Natl. Acad. Sci., 99(2):685-690, 2002.

14. Margaret S. Cheung and D. Thirumalai. Nanopore-protein interactions dramatically alter stability and yield of the native state in restricted spaces. J Mol Biol, 357(2):632-643, 2006.

15. Samuel S. Cho, Yaakov Levy, and Peter G. Wolynes. P versus q: Structural reaction coordinates capture protein folding on smooth landscapes. Proc Nat Acad Sci USA, 103:586-591, 2006.

16. C. Clementi and S. S. Plotkin. The effects of nonnative interactions on protein folding rates: Theory and simulation. Protein Sci, 13:1750-1766, 2004.

17. Payel Das, Mark Moll, Hernan Stamati, Lydia E. Kavraki, and Cecilia Clementi. Low-dimensional, free-energy landscapes of protein-folding reactions by nonlinear dimensionality reduction. Proc Nat Acad Sci USA, 103(26):9885-9890, 2006.

18. Nikolay V. Dokholyan, Lewyn Li, Feng Ding, and Eugene I. Shakhnovich. Topological determinants of protein folding. Proc. Nat. Acad. Sci. USA, 99(13):8637-8641, 2002.

19. R. Du, V. S. Pande, A. Yu. Grosberg, T. Tanaka, and E. S. Shakhnovich. On the transition coordinate for protein folding. J Chem Phys, 108:334-350, 1998.

20. A. R. Fersht. Structure and mechanism in protein science. W. H. Freeman and Co., New York, first edition, 1999.

21. O. V. Galzitskaya and A. V. Finkelstein. A theoretical search for folding/unfolding nuclei in threedimensional protein structures. Proc Nat Acad Sci USA, 96:11299-11304, 1999.

22. A. E. García. Large-amplitude nonlinear motions in proteins. Phys Rev Lett, 68:2696-2699, 1992.

23. Angel E. Garcia and Jose N. Onuchic. Folding a protein in a computer: An atomic description of the folding/unfolding of protein A. Proc. Natl. Acad. Sci., 100(24):13898-13903, 2003. 
24. John Gregory and Cantian Lin. An unconstrained calculus of variations formulation for generalized optimal control problems and for the constrained problem of bolza. J. Math. Anal. Appl., 187:826841, 1994.

25. John Gregory and Cantian Lin. Constrained Optimization in the Calculus of Variations and Optimal Control Theory. Springer, New York, first edition, 2007.

26. G. Hummer, A. E. García, and S. Garde. Conformational diffusion and helix formation kinetics. Phys Rev Lett, 85:2637-2640, 2000.

27. G. Hummer, A. E. García, and S. Garde. Helix nucleation kinetics from molecular simulations in explicit solvent. Proteins, 42:77-84, 2001.

28. John Karanicolas and III Brooks, Charles L. The origins of asymmetry in the folding transition states of protein L and protein G. Protein Sci, 11(10):2351-2361, 2002.

29. Sergei V. Krivov, Stefanie Muff, Amedeo Caflisch, and Martin Karplus. One-dimensional barrierpreserving free-energy projections of a $\beta$-sheet miniprotein: New insights into the folding process. Journal of Physical Chemistry B, 112(29):8701-8714, 2008.

30. R. D. Levine and R. B. Bernstein, editors. Molecular reaction dynamics and chemical reactivity. Clarendon Press, Oxford, 1987.

31. M. Lindberg, Jeanette Tangrot, and M. Oliveberg. Complete change of the protein folding transition state upon circular permutation. Preprint, 2002.

32. Ao Ma and Aaron R. Dinner. Automatic method for identifying reaction coordinates in complex systems. J. Phys. Chem. B, 109(14):6769-6779, 2005.

33. Ali R. Mohazab and Steven S. Plotkin. Minimal folding pathways for coarse-grained biopolymer fragments. Biophys. J. (to appear) BIOFAST 19 September (2008).

34. Ali R. Mohazab and Steven S. Plotkin. Minimal distance transformations between links and polymers: principles and examples. J. Phys. Cond. Mat., 20:244133, 2008.

35. V. Munoz and W. A. Eaton. A simple model for calculating the kinetics of protein folding from three-dimensional structures. Proc. Natl Acad. Sci. USA, 96:11311-11316, 1999.

36. H. Nymeyer, N. D. Socci, and J. N. Onuchic. Landscape approaches for determining the ensemble of folding transition states: Success and failure hinge on the degree of minimal frustration. Proc. Natl Acad. Sci. USA, 97:634-639, 2000.

37. L. Onsager. Initial recombination of ions. Phys. Rev., 54:554-557, 1938.

38. J. N. Onuchic, Z. Luthey-Schulten, and P. G. Wolynes. Theory of protein folding: The energy landscape perspective. Annu Rev Phys Chem, 48:545-600, 1997.

39. S. S. Plotkin. Speeding protein folding beyond the gō model: How a little frustration sometimes helps. Proteins: Struct. Funct. Genet., 45:337-345, 2001.

40. S. S. Plotkin. Generalization of distance to higher dimensional objects. Proc. Natl Acad. Sci. USA, 104(38):14899-14904, 2007.

41. S. S. Plotkin and J. N. Onuchic. Investigation of routes and funnels in protein folding by free energy functional methods. Proc. Natl Acad. Sci. USA, 97:6509-6514, 2000. 
42. S. S. Plotkin and J. N. Onuchic. Structural and energetic heterogeneity in protein folding i: Theory. J. Chem. Phys., 116(12):5263-5283, 2002.

43. S. S. Plotkin and J. N. Onuchic. Understanding protein folding with energy landscape theory i: Basic concepts. Quart. Rev. Biophys., 35(2):111-167, 2002.

44. S. S. Plotkin and J. N. Onuchic. Understanding protein folding with energy landscape theory ii: Quantitative aspects. Quart. Rev. Biophys., 35(3):205-286, 2002.

45. S. S. Plotkin, J. Wang, and P. G. Wolynes. Statistical mechanics of a correlated energy landscape model for protein folding funnels. J Chem Phys, 106:2932-2948, 1997.

46. S. S. Plotkin and P. G. Wolynes. Non-markovian configurational diffusion and reaction coordinates for protein folding. Phys. Rev. Lett., 80:5015-5018, 1998.

47. S. S. Plotkin and P. G. Wolynes. Buffed energy landscapes: Another solution to the kinetic paradoxes of protein folding. Proc. Natl Acad. Sci. USA, 100(8):4417-4422, 2003.

48. L. S. Pontryagin, V. G. Boltyanskii, R. V. Gamkrelidze, and E. F. Mishchenko. The mathematical theory of optimal processes. Wiley Interscience, New York and London, 1962.

49. J. J. Portman, S. Takada, and P. G. Wolynes. Microscopic theory of protein folding rates. I. Fine structure of the free energy profile and folding routes from a variational approach. J. Chem. Phys., 114:5069-5081, 2001.

50. J. J. Portman, S. Takada, and P. G. Wolynes. Microscopic theory of protein folding rates. II. Local reaction coordinates and chain dynamics. J. Chem. Phys., 114:5082-5096, 2001.

51. C.D. Snow, H. Nguyen, V.S. Pande, and M. Gruebele. Absolute comparison of simulated and experimental protein-folding dynamics. Nature, 420:102-106, 2002.

\section{Tables}

Table 1: $\mathcal{D} / N$ (in units of link length squared) between the aligned structures in figure 11, Each of the 4 columns represents the structural pairs for the cost function labelled. For example, column 3 gives $\mathcal{D} / N$ for structural pairs in figure 11 aligned using MRSD.

\begin{tabular}{c|c|c|c|c} 
& \multicolumn{4}{|c}{ Alignment cost function } \\
$N$ & $\mathcal{D}$ & $\mathcal{D}_{1}$ & MRSD & RMSD \\
\hline 4 & 0.785 & 0.785 & 0.785 & 0.822 \\
6 & 1.391 & 1.415 & 1.473 & 1.419 \\
8 & 1.974 & 1.983 & 2.085 & 2.014 \\
10 & 2.559 & 2.574 & 2.654 & 2.615 \\
12 & 3.127 & 3.158 & 3.197 & 3.216 \\
14 & 3.674 & 3.705 & 3.726 & 3.817 \\
16 & 4.207 & 4.235 & 4.247 & 4.418 \\
18 & 4.732 & 4.769 & 4.762 & 5.019 \\
20 & 5.252 & 5.294 & 5.272 & 5.620 \\
22 & 5.767 & 5.802 & 5.783 & 6.221
\end{tabular}


Table 2: MRSD (in units of link length) between the aligned structures in figure 11 using the four cost functions we considered. For example, column 1 gives MRSD for structural pairs in figure 11 aligned using the distance $\mathcal{D}$.

\begin{tabular}{c|c|c|c|c} 
& \multicolumn{4}{|c}{ Alignment cost function } \\
$N$ & $\mathcal{D}$ & $\mathcal{D}_{1}$ & MRSD & RMSD \\
\hline 4 & 0.707 & 0.707 & 0.707 & 0.809 \\
6 & 1.375 & 1.393 & 1.337 & 1.412 \\
8 & 1.961 & 1.960 & 1.899 & 2.008 \\
10 & 2.547 & 2.545 & 2.436 & 2.610 \\
12 & 3.062 & 3.108 & 2.959 & 3.211 \\
14 & 3.575 & 3.675 & 3.475 & 3.813 \\
16 & 4.081 & 4.004 & 3.987 & 4.414 \\
18 & 4.585 & 4.506 & 4.495 & 5.015 \\
20 & 5.088 & 5.008 & 5.002 & 5.616 \\
22 & 5.591 & 5.511 & 5.508 & 6.218
\end{tabular}




\section{Figures}

Figure 1: Order parameters do not always correlate with kinetic proximity. Structure $A$ above is more native-like according to the fraction of native contacts, while structure $B$ is more native-like according to $R M S D$, and is also closer kinetically to the native structure.

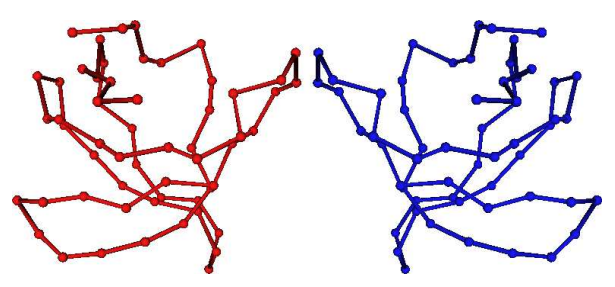

Figure 2: Native structure of SH3 (right) and its mirror image. Although dissimilar by RMSD, biologically nonfunctional, and disallowed by true dihedral potentials, this structure has a $Q=1$, because native contacts remain intact after mirroring transformations. 


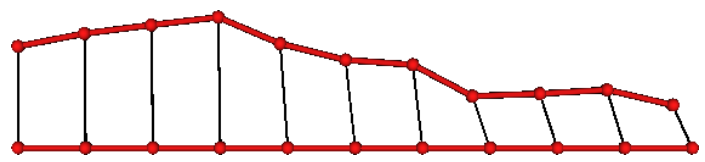

Figure 3: The MRSD is the average length of the black like segments between corresponding residues of the initial and final configuration.

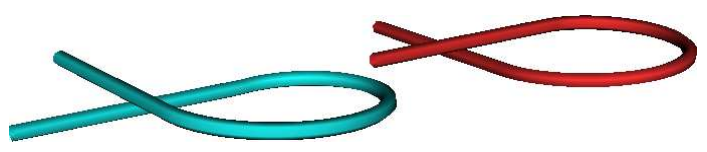

Figure 4: The MRSD and RMSD between the two curves are close to zero (the curves in this figure are displaced for better viewing but should be imagined to be superposed). But because the curve cannot pass through itself, in order to undergo the transformation one leg must undergo relatively large amplitude motions to travel from one conformation to another. This results in a non-zero distance between the conformations by accurate metrics which can account for non-crossing.

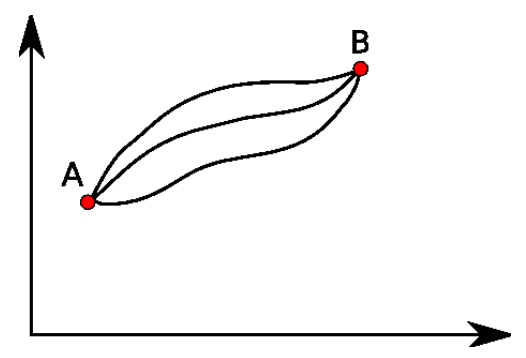

Figure 5: Distance between the two points $A$ and $B$ is the minimum length of the the curve connecting the two points.

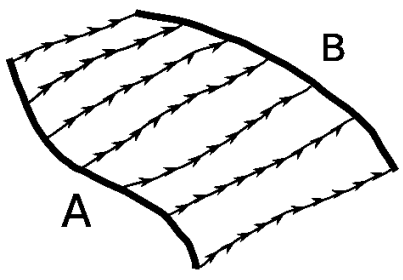

Figure 6: The distance $\mathcal{D}_{A B}$ is the accumulation of how much every part of the contour defining the space curve moves in the transformation between two conformations $A$ and $B$.

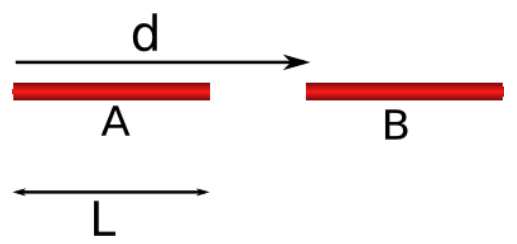

Figure 7: The line segment $A$ is displaced by $d$ along itself, to $B$. The soap film area $A_{\text {soap }}$ between the two segments is 0 . But the distance $\mathcal{D}_{A B}=L d$ 


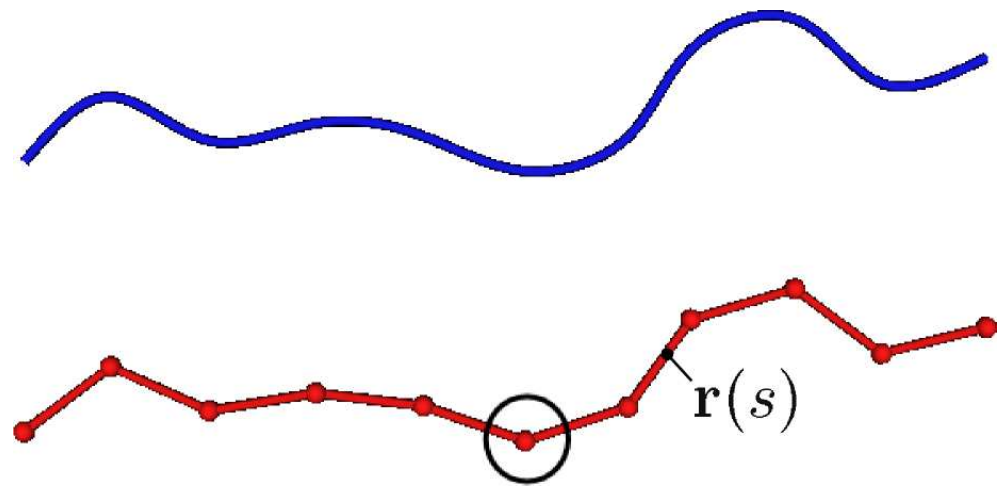

Figure 8: The lower curve is a discretized version of the upper one. After discretization the PDE for the upper curve becomes a set of $N$ coupled ODE's for the $N$ residues in the lower chain (A sample residue is marked with a circle).

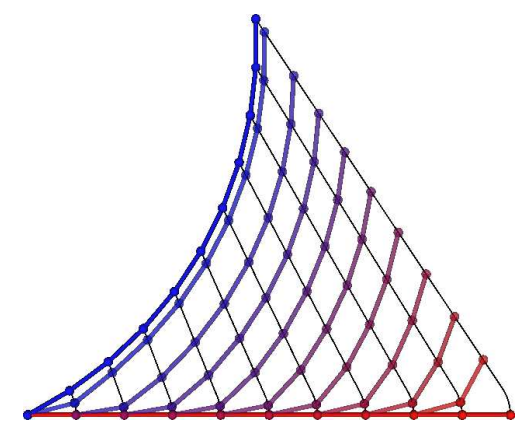

(a)

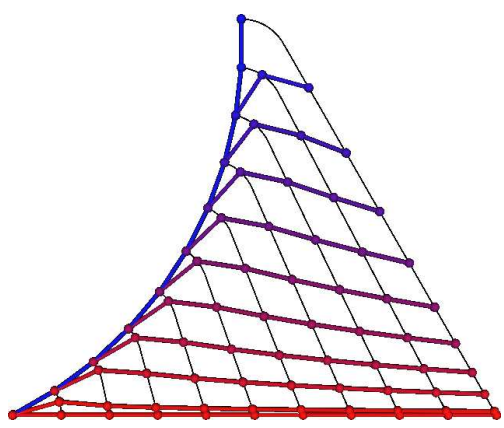

(b)

Figure 9: Minimal and sub-minimal transformations between a straight line and a quarter circle (see text for description). For the left transformation $\mathcal{D}=45.793$ and for the right one $\mathcal{D}=46.278$

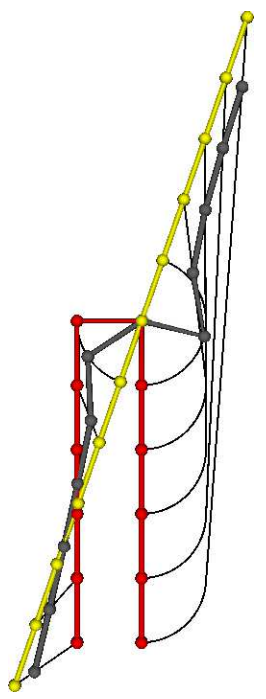

(a)

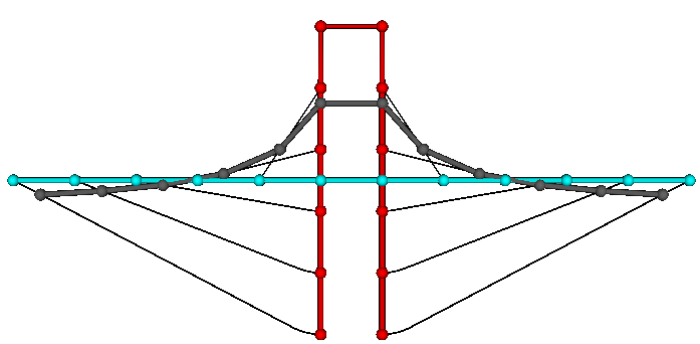

(b)

Figure 10: (color) $\mathcal{D}$ minimizing transformations for MRSD aligned (yellow) and RMSD (cyan) aligned hairpins. Intermediate state is shown in grey. The distances for each transformation, in units of link length squared, are 3.20 for MRSD-aligned and 3.22 for RMSD-aligned structures. 


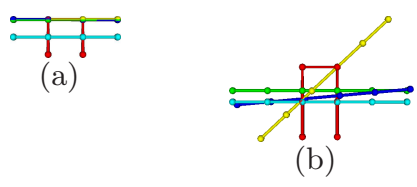

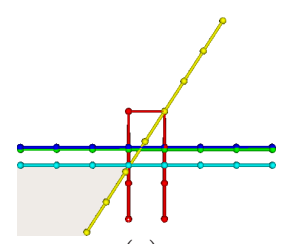

(c)

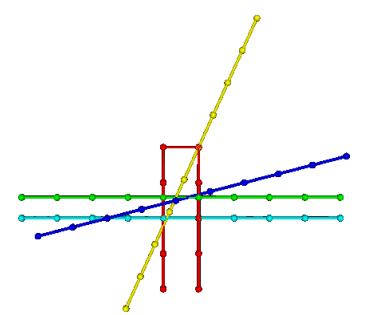

(d)

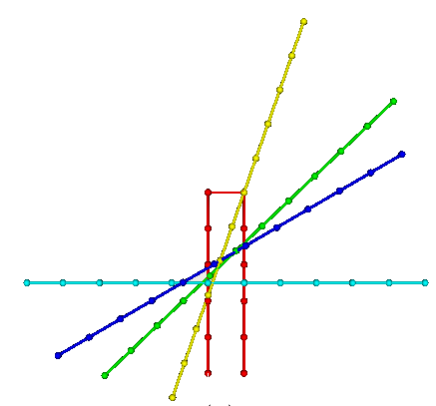

(e)

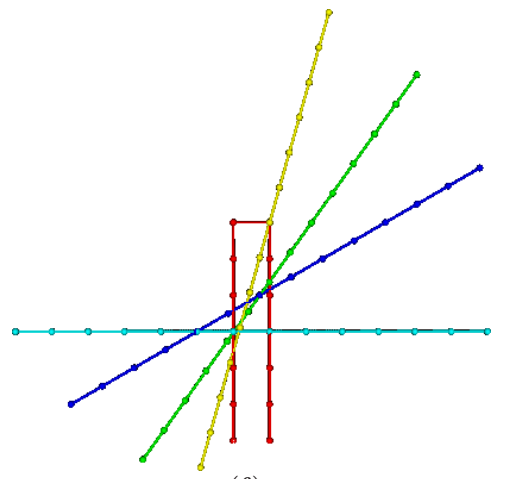

(f)

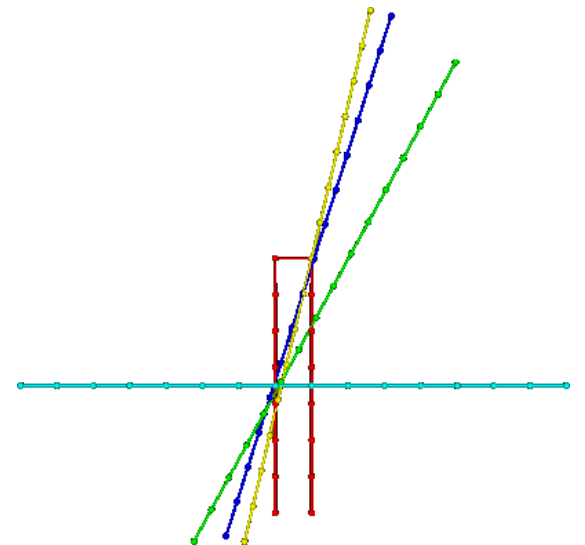

(g)

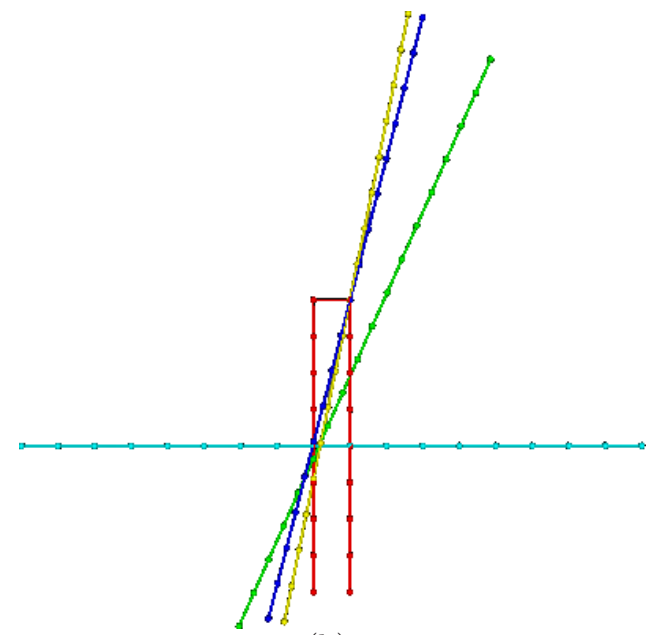

(h)

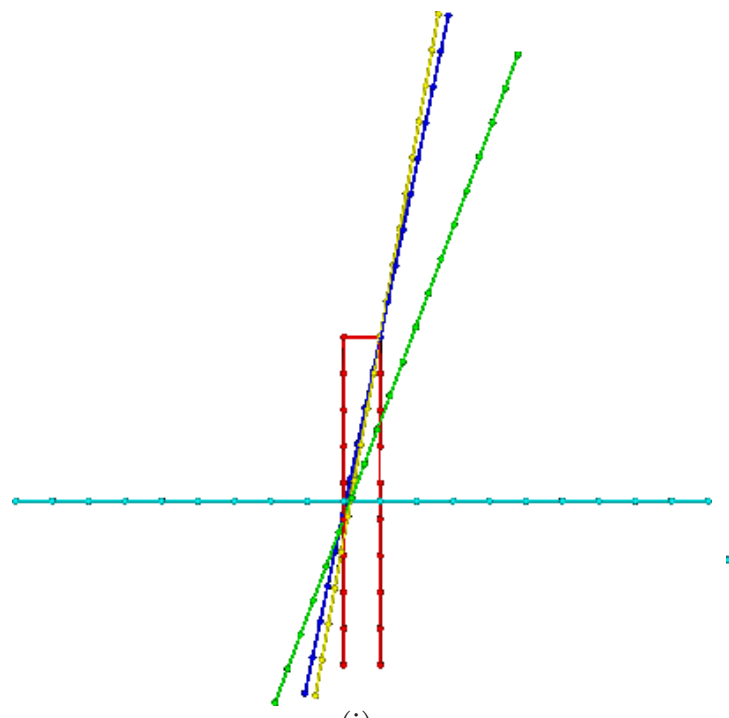

(i)

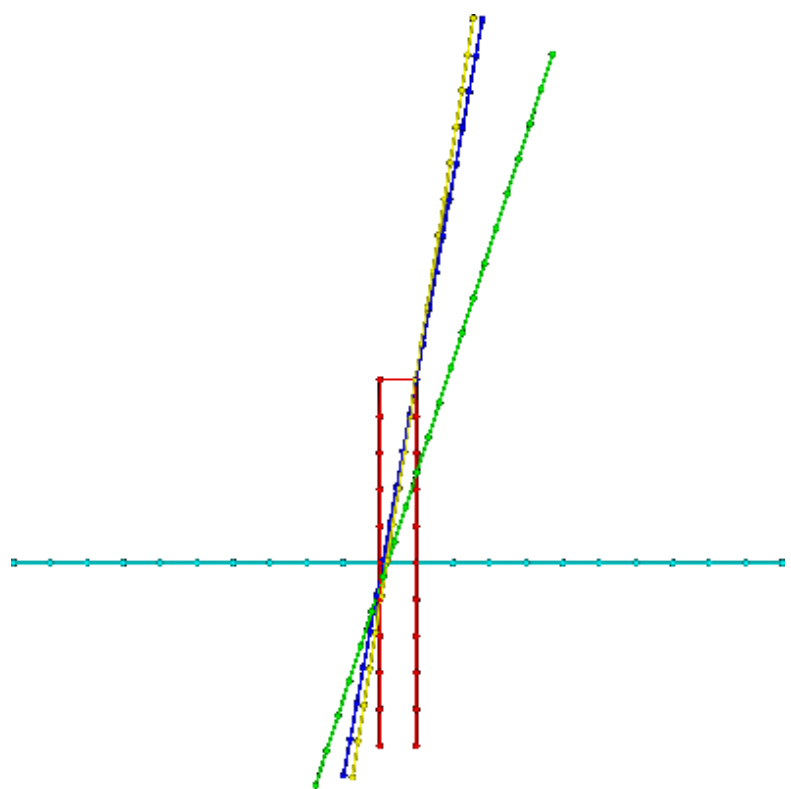

(j)

Figure 11: (color) Alignments with different cost functions. The Hairpin is shown in red. $\mathcal{D}$ alignment in green, $\mathcal{D}_{1}$ in blue, MRSD in yellow, and RMSD in cyan 


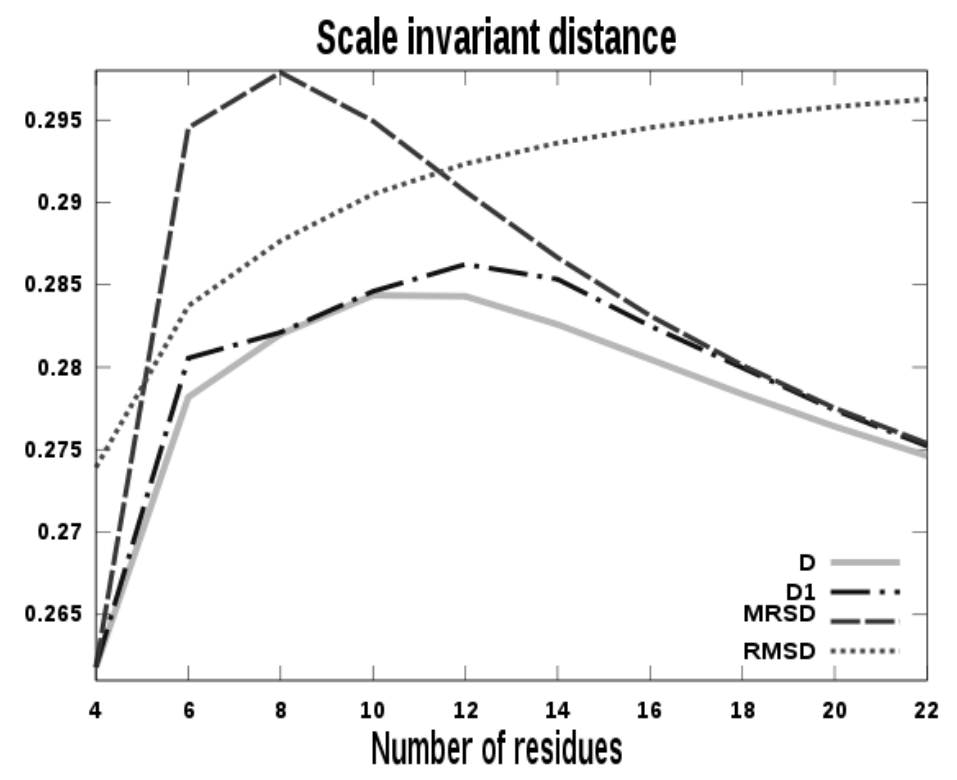

Figure 12: Scale invariant distance resulting from different alignments with different cost functions 